\title{
Clinical Significance of an HPV DNA Chip Test with Emphasis on HPV-16 and/or HPV-18 Detection in Korean Gynecological Patients
}

\author{
Min-Kyung Yeo ${ }^{1,2} \cdot$ Ahwon Lee ${ }^{1}$ \\ Soo Young Hur ${ }^{3}$. Jong Sup Park \\ ${ }^{1}$ Department of Hospital Pathology, College \\ of Medicine, The Catholic University of Korea, \\ Seoul; ' ${ }^{2}$ epartment of Pathology, Chungnam \\ National University School of Medicine, Daejeon; \\ ${ }^{3}$ Department of Obstetrics and Gynecology, \\ Seoul St. Mary's Hospital, College of Medicine, \\ The Catholic University of Korea, Seoul, Korea \\ Received: January 26, 2016 \\ Revised: April 6, 2016 \\ Accepted: May 9, 2016 \\ Corresponding Author \\ Ahwon Lee, MD \\ Department of Hospital Pathology, \\ College of Medicine, The Catholic University \\ of Korea, 222 Banpo-daero, Seocho-gu, \\ Seoul 06591, Korea \\ Tel: +82-2-2258-1621 \\ Fax: +82-2-2258-1627 \\ E-mail: klee@catholic.ac.kr
}

\begin{abstract}
Background: Human papillomavirus (HPV) is a major risk factor for cervical cancer. Methods: We evaluated the clinical significance of the HPV DNA chip genotyping assay (MyHPV chip, Mygene Co.) compared with the Hybrid Capture 2 ( $\mathrm{HC} 2)$ chemiluminescent nucleic acid hybridization kit (Digene Corp.) in 867 patients. Results: The concordance rate between the MyHPV chip and HC2 was $79.4 \%$ (kappa coefficient, $\kappa=0.55$ ). The sensitivity and specificity of both HPV tests were very similar (approximately $85 \%$ and $50 \%$, respectively). The addition of HPV result (either MyHPV chip or HC2) to cytology improved the sensitivity (95\%, each) but reduced the specificity (approximately $30 \%$, each) compared with the HPV test or cytology alone. Based on the MyHPV chip results, the odds ratio (OR) for $\geq$ high-grade squamous intraepithelial lesions (HSILs) was 9.9 in the HPV-16/18 (+) group and 3.7 in the non-16/18 high-risk (HR)-HPV (+) group. Based on the HC2 results, the OR for $\geq$ HSILs was 5.9 in the HR-HPV (+) group. When considering only patients with cytological diagnoses of "negative for intraepithelial lesion or malignancy" and "atypical squamous cell or atypical glandular cell," based on the MyHPV chip results, the ORs for $\geq$ HSILs were 6.8 and 11.7, respectively, in the HPV-16/18 (+) group. Conclusions: The sensitivity and specificity of the MyHPV chip test are similar to the HC2. Detecting HPV-16/18 with an HPV DNA chip test, which is commonly used in many Asian countries, is useful in assessing the risk of high-grade cervical lesions.
\end{abstract}

Key Words: Human papillomavirus; DNA chip; Hybrid capture 2; Cervical intraepithelial neoplasia; Cervical carcinoma
Human papillomavirus (HPV) is a DNA tumor virus that is an essential causative factor for cervical cancer. Persistent infection, particularly with high-risk (HR) HPV (HR-HPV) genotypes, plays a major role in the progression of precancerous cervical lesions to invasive cancer. ${ }^{1}$ The incidence rate of high-grade cervical lesions is reported to be higher in patients infected with HPV-16 and/or HPV-18 (HPV-16/18) than in patients with other HR-HPV strains, ${ }^{2-5}$ and HPV-16 and HPV-18 cause $71 \%$ of HPV infections in cervical cancer patients. ${ }^{6,7}$

Although cytology in cervical cancer screening has been successful, it has several limitations, including low sensitivity and reproducibility. Addition of HPV DNA test to the cytology test, so-called co-test, is the preferred screening method for prevention and early detection of cervical cancer according to the 2012 American Cancer Society screening guidelines. ${ }^{8}$ For the first time, these recommendations have adopted HPV 16/18 genotyping in the management of women with positive HPV tests and negative cytology. Specifically, these women should under- go subsequent HPV-16/18 genotyping or repeat co-testing in 12 months. ${ }^{8}$

The Hybrid Capture 2 (HC2) chemiluminescent nucleic acid hybridization kit (Digene Corp., Gaithersburg, MA, USA) is a frequently used method to detect HR-HPV and is approved by the United States Food and Drug Administration (U.S. FDA). The kit detects $13 \mathrm{HR}-\mathrm{HPV}$ types and reports results as either HR-HPV (+) or HR-HPV (-).

The HPV DNA chip test (MyHPV chip, Mygene Co., Seoul, Korea) identifies $15 \mathrm{HR}$ and nine low-risk HPV types and is a commercial HPV DNA genotyping tool used in Korea and other Asian countries. Furthermore, the MyHPV chip kit is approved by the Korean Food and Drug Administration (K-FDA). However, no previous study has assessed the association between HPV-16/18 detected using the MyHPV chip and the risk of developing high-grade cervical lesions.

In this study, we compared the clinical performance of the MyHPV chip and HC2 tests using a histological cut-off for high- 
grade squamous intraepithelial lesions (HSILs).

\section{MATERIALS AND METHODS}

\section{Study subjects}

This study was approved by the Institutional Review Board of The Catholic University of Korea, Seoul St. Mary's Hospital, and complied with the tenets of the Declaration of Helsinki. We retrospectively reviewed the pathology archive database records of 867 consecutive gynecological patients treated at Seoul St. Mary's Hospital, from January 2006 to December 2009, for whom cervical cytology and HPV test results from both the HC2 and MyHPV chip were available and confirmed by histological examination. The majority of patients were referred to our institution due to abnormal results in routine cervical examinations, and thus the patients underwent cervical cytology, HPV test (HC2 to confirm HPV infection and MyHPV chip to determine infected HPV genotype), and colposcopic examinations followed by tissue biopsy if necessary. The cervical cytology, HC2 test, and MyHPV chip tests were simultaneously performed on the same sample in most cases. Otherwise, we selected cytology that was performed within 2 weeks prior to HPV testing. We classified cervical cytology according to the 2001 Bethesda System for Reporting Cervical Cytology. ${ }^{9}$ The diagnoses of all included patients were pathologically confirmed by biopsy, conization and/ or hysterectomy within 3 months of cervical cytology and HPV testing. The patients were divided based on age as follows: $\leq 29$ years, 178 patients (20.5\%); 30-39 years, 281 patients (32.4\%); 40-49 years, 243 patients (28.0\%); 50-59 years, 109 patients (12.6\%); and $\geq 60$ years, 56 patients (6.5\%); the mean age was 40 years.

\section{HPV genotyping using the MyHPV chip test}

The MyHPV chip test contains probes for 15 HR (HPV-16, $-18,-31,-33,-35,-39,-45,-51,-52,-53,-56,-58,-59,-66$, and -68) and 9 low-risk HPV types (HPV 6, 11, 34, 40, 42, 43, 44, 54, and 70). The MyHPV chip test was performed according to the manufacturer's instructions under the supervision of Dr. A. Lee (pathologist with a specialty in gynecopathology and molecular pathology). The cervical DNA was isolated from specimens and amplified using polymerase chain reaction (PCR) with consensus $\mathrm{GP}^{+} / \mathrm{GP}^{+}$primers. Beta-globin was amplified as an internal control. The PCR product $(5 \mu \mathrm{L})$ was subjected to $2.5 \%$ agarose gel electrophoresis. Twenty-four type-specific 30mer oligonucleotide probes containing an amine group at the $5^{\prime}$ terminus were immobilized onto a slide glass chip. The PCR products were labelled with Cy5-dUTP, denatured, mixed with hybridization solution, and incubated on the DNA chip. The hybridized HPV DNA was visualized using a DNA chip scanner (ScanArray LITE, GSI Lumonics Inc., Bedford, MA, USA).

Clearly visualized double-positive spots for a specific HPV type were considered "specific HPV-positive." Samples negative on the chip scanner but positive for the 150-bp HPV-specific band using gel electrophoresis were interpreted as "negative for 15 HR-HPV and nine low-risk HPV types but positive for other HPV types." A lack of visualized spots and samples negative for the 150-bp HPV-specific band using gel electrophoresis were considered "HPV-negative."

\section{HC2 DNA hybridization assay}

The HC2 test was performed according to the manufacturer's instructions as previously described. ${ }^{10}$ In brief, the specimens for the HC2 test were denatured and hybridized with RNA probes to detect 13 HR-HPV types (HPV-16, -18, $-31,-33,-35,-39$, $-45,-51,-52,-56,-58,-59$, and -68 ) in a microplate format. These hybrids react with multiple antibody conjugates and are quantified based on an amplified chemiluminescent signal. Relative light units/positive control values $\geq 1.00$ were considered "positive," whereas values $<1.00$ were considered "negative."

\section{Statistical analysis}

The concordance rates of the MyHPV chip and HC2 were evaluated using the kappa coefficient ( $\kappa$ ) with $95 \%$ confidence intervals (CIs). The sensitivity, specificity, positive predictive value (PPV), and negative predictive value (NPV) of the $\mathrm{MyH}-$ PV chip and HC2 for $\geq$ HSILs were determined using standard statistical tests. The age-adjusted odds ratios (ORs) for $\geq$ HSILs with 95\% CI were evaluated for different categories of MyHPV chip and $\mathrm{HC} 2$ results with a binary logistic regression using the SPSS ver. 22.0 software (IBM Co., Armonk, NY, USA). To analyze the MyHPV chip results, patients with single or multiple samples positive for $15 \mathrm{HR}$ genotypes were categorized as HR-HPV (+). Patients with HPV-16 (+) and/or HPV-18 (+) were categorized as HPV-16/18 (+), irrespective of the presence of any other HPV genotypes. Patients with HPV-16 (-) and HPV-18 (-), 13 other HR-HPV (+) with or without low-risk HPV $(+)$ or "HPV-other types" $(+)$ were categorized as non16/18 HR-HPV (+). Patients with low-risk HPV (+), "HPVother types" (+) or HPV (-) were categorized as HR-HPV (-). In cases of multiple infections, patients were classified based on the HPV genotypes associated with a higher risk of invasive cancer. For example, a patient with HPV-16 (+) and HPV-31 (+) 
was allocated to the HPV-16/18 (+) group.

\section{RESULTS}

\section{HPV, cytology, and histology test results}

Among 867 patients, 575 (66.3\%) were HR-HPV (+) according to the MyHPV chip test, 540 (62.3\%) were HR-HPV $(+)$ according to the HC2 test, and 545 (62.9\%) were classified as atypical squamous cells of undetermined significance (ASCUS) or worse ( $\geq$ ASCUS) cytology. The following cytological diagnoses were made for 867 patients: 322 patients were negative for intraepithelial lesion or malignancy (NILM), 138 patients exhibited ASCUS, six patients exhibited atypical glandular cells (AGC), 30 patients exhibited atypical squamous cells for which HSILs (ASC-H) cannot be excluded, 183 patients exhibited low-grade squamous intraepithelial lesions (LSILs), 123 patients exhibited HSILs, including carcinoma in situ, and 65 patients exhibited invasive cancer, including squamous cell carcinoma and adenocarcinoma (Table 1). The overall prevalence rates of histology confirmed HSIL or worse ( $\geq$ HSIL) according to the cytological diagnosis of NILM, ASCUS, LSIL, ASC-H, HSIL, and invasive cancer were $12.4 \%, 25.4 \%, 24.0 \%, 60.0 \%, 83.7 \%$, and $98.4 \%$, respectively. The overall prevalence rates of $\geq \mathrm{HSIL}$ according to the MyHPV chip test for HPV-16/18 (+), non16/18 HR-HPV (+), and HR-HPV (-) were $58.1 \%, 36.0 \%$, and $13.0 \%$, respectively. The overall prevalence rates of $\geq \mathrm{HSIL}$ according to the HC2 test for HR-HPV (+) and HR-HPV (-) were $48.3 \%$ and $13.5 \%$, respectively (Tables 1, 2).

\section{Clinical performance of the cytology, the MyHPV chip and the $\mathrm{HC} 2$}

The concordance rate between the MyHPV chip test and the HC2 test was 79.4\% (688/867), with a $\mathrm{K}$-value of 0.551 (Table 3). Among 272 cases with HPV-16/18 (+) based on the MyHPV

Table 1. Histology results according to cytology, HPV DNA chip results, ${ }^{a}$ and HC2 results

\begin{tabular}{|c|c|c|c|c|c|}
\hline Histology & Cenvicitis ( $n=241$ ) & $\operatorname{LSIL}(n=321)$ & HSIL $(n=210)$ & Cancer $(n=95)$ & Total $(n=867)$ \\
\hline \multicolumn{6}{|l|}{ Cytology } \\
\hline NILM & $161(66.8)$ & $121(37.7)$ & $29(13.8)$ & $11(11.6)$ & 322 \\
\hline ASCUS & $28(11.6)$ & 75 (23.4) & $31(14.8)$ & $4(4.2)$ & 138 \\
\hline ASC-H & $3(1.2)$ & $9(2.8)$ & $14(6.7)$ & $4(4.2)$ & 30 \\
\hline AGC & $5(2.1)$ & $0(0)$ & $1(0.5)$ & $0(0)$ & 6 \\
\hline LSIL & 39 (16.2) & $100(31.2)$ & $42(20)$ & $2(2.1)$ & 183 \\
\hline HSIL & $4(1.7)$ & $16(5.0)$ & $86(41.0)$ & $17(17.9)$ & 123 \\
\hline Cancer & $1(0.4)$ & $0(0)$ & $7(3.3)$ & $57(60.0)$ & 65 \\
\hline \multicolumn{6}{|l|}{ HPV DNA chip } \\
\hline HR-HPV (-) & $133(55.2)$ & $121(37.7)$ & $27(12.9)$ & $11(11.6)$ & 292 \\
\hline Non-16/18 HR-HPV (+) & $67(27.8)$ & $127(39.6)$ & $84(40.0)$ & $25(26.3)$ & 303 \\
\hline HPV-16/18 (+) & $41(17.0)$ & $73(22.7)$ & $99(47.1)$ & $59(62.1)$ & 272 \\
\hline \multicolumn{6}{|l|}{ HPV HC2 } \\
\hline HR-HPV (-) & $155(64.3)$ & $128(39.9)$ & 28 (13.3) & $16(16.8)$ & 327 \\
\hline HR-HPV (+) & $86(35.7)$ & $193(60.1)$ & $182(86.7)$ & 79 (83.2) & 540 \\
\hline
\end{tabular}

HPV, human papillomavirus; HC2, Hybrid Capture 2; LSIL, low-grade squamous intraepithelial lesion; HSIL, high-grade squamous intraepithelial lesion; NILM, negative for intraepithelial lesion or malignancy; ASCUS, atypical squamous cells of undetermined significance; ASC-H, atypical squamous cells cannot exclude HSIL; AGC, atypical glandular cells; HR, high-risk.

aHPV-HR (-) includes HPV (-) or low-risk HPV (+) or "HPV-other types" (+); non-16/18 HR-HPV (+) includes HPV-16 (-) and HPV-18 (-), 13 other HR-HPV (+) with or without low-risk HPV (+) or "HPV-other types" (+); HPV-16/18 (+) includes HPV-16 (+) and/or HPV-18 (+), with or without any other HPV genotypes present.

Table 2. Age-adjusted odds ratio for $\geq \mathrm{HSIL}$ histology in each HPV group

\begin{tabular}{|c|c|c|c|c|c|}
\hline & Total & $\geq \mathrm{HSIL}$ & Odds ratio & $95 \% \mathrm{Cl}$ & $p$-value \\
\hline \multicolumn{6}{|l|}{ HPV DNA chipa } \\
\hline HR-HPV (-) & 292 & $38(13.0)$ & & & \\
\hline Non-16/18 HR-HPV (+) & 303 & $109(36.0)$ & 3.739 & $2.448-5.709$ & .000 \\
\hline HPV-16/18 (+) & 272 & $158(58.1)$ & 9.874 & $6.418-15.190$ & .000 \\
\hline \multicolumn{6}{|l|}{ HPV HC2 } \\
\hline HR-HPV (-) & 327 & $44(13.5)$ & & & \\
\hline HR-HPV (+) & 540 & 261 (48.3) & 5.914 & $4.102-8.527$ & .000 \\
\hline
\end{tabular}

2HSIL, high-grade squamous intraepithelial lesion or worse; HPV, human papillomavirus; $\mathrm{Cl}$, confidence interval; HR, high-risk.

aHPV-HR (-) includes HPV (-) or low-risk HPV (+) or "HPV-other types" (+); non-16/18 HR-HPV (+) includes HPV-16 (-) and HPV-18 (-), 13 other HR-HPV (+) with or without low-risk HPV (+) or "HPV-other types" (+); HPV-16/18 (+) includes HPV-16 (+) and/or HPV-18 (+), with or without any other HPV genotypes present. 
chip test, 229 cases (84.2\%) were also HR-HPV (+) based on HC2 test. The sensitivity, specificity, PPV, and NPV of the cytology, HC2 and MyHPV chip for $\geq$ HSIL histology were evaluated and found similar among the tests (Table 4). The clinical performance of cytology and HC2 as well as cytology and the MyHPV chip were then evaluated (Table 4). The addition of either HPV test (HC2 or HPV DNA) to cytology for detecting $\geq$ HSIL histology improved the sensitivity and NPV, but reduced the specificity and PPV compared with the HPV test or cytology alone.

Table 3. Comparison between HPV DNA chip results ${ }^{\mathrm{a}}$ and HC2 results

\begin{tabular}{lcccc}
\hline & \multicolumn{3}{c}{ HPV DNA chip } & \\
\cline { 2 - 4 } HC2 & Negative & $\begin{array}{c}\text { Non-16/18 } \\
\text { HR-HPV }(+)\end{array}$ & HPV-16/18 (+) & \\
\hline Negative & $220(75.3)$ & $64(21.1)$ & $43(15.8)$ & 327 \\
Positive & $72(24.7)$ & $239(78.9)$ & $229(84.2)$ & 540 \\
Total & 292 & 303 & 272 & 867 \\
\hline
\end{tabular}

HPV, human papillomavirus; HC2, Hybrid Capture 2; HR, high-risk. aHR-HPV (-) includes HPV (-) or low-risk HPV (+) or "HPV-other types"(+); non-16/18 HR-HPV (+) includes HPV-16 (-) and HPV-18 (-), 13 other HRHPV $(+)$ with or without low-risk HPV (+) or "HPV-other types" (+); HPV16/18 (+) includes HPV-16 (+) and/or HPV-18 (+), with or without any other HPV genotypes present.

\section{ORs for $\geq$ HSILs according to the MyHPV chip and HC2 results}

We calculated the ORs for $\geq$ HSILs based on the MyHPV chip and HC2 results. The MyHPV chip results were categorized as HPV-16/18 (+), non-16/18 HR-HPV (+), and HR-HPV (-) groups. The age-adjusted ORs for $\geq \mathrm{HSIL}$ were $9.9(95 \% \mathrm{CI}, 6.4$ to 15.2$)$ in the HPV-16/18 (+) group and 3.7 (95\% CI, 2.4 to 5.7$)$ in the non-16/18 HR-HPV (+) group. Based on HC2 results, the age-adjusted OR for $\geq \mathrm{HSIL}$ was $5.9(95 \% \mathrm{CI}, 4.1$ to 8.5$)$ in the HR-HPV (+) group (Table 2). A further subgroup analysis was performed among patients with cytological diagnoses of "NILM" and "atypical squamous cells (ASC) or AGC" (Table 5). Regarding the cytology of "NILM" patients, based on MyHPV chip results, the age-adjusted OR for $\geq$ HSIL in the HPV-16/18 (+) group was 6.8 (95\% CI, 2.1 to 21.6), but the OR for $\geq \mathrm{HSIL}$ in the non-16/18 HR-HPV (+) group did not significantly differ from the OR in the HR-HPV (-) group. Based on the HC2 results, the age-adjusted OR for $\geq \mathrm{HSIL}$ was $5.9(95 \% \mathrm{CI}, 2.9$ to 12.2) in the HR-HPV (+) group (Table 5). Regarding the cytology of "ASC or AGC" patients, based on the MyHPV chip results, the age-adjusted OR for $\geq \mathrm{HSIL}$ was 11.7 (95\% CI, 4.1 to 33.5) in the HPV-16/18 (+) group, but the OR for $\geq$ HSIL in the

Table 4. Clinical performance of cytology, HPV DNA chip test, and HC2 test

\begin{tabular}{lccccc}
\hline & Cytology & HPV DNA chip & HC2 & Cytology+HC2 & Cytology+HPV DNA chip \\
\hline Sensitivity & 0.869 & 0.875 & 0.856 & 0.954 & 0.951 \\
Specificity & 0.502 & 0.452 & 0.504 & 0.379 & 0.304 \\
PPV & 0.486 & 0.464 & 0.483 & 0.455 & 0.426 \\
NPV & 0.876 & 0.87 & 0.865 & 0.938 & 0.919 \\
\hline
\end{tabular}

HPV, human papillomavirus; HC2, Hybrid Capture 2; PPV, positive predictive value; NPV, negative predictive value.

Table 5. Age-adjusted odds ratio for $\geq H S I L$ histology in each HPV group exhibiting "NILM" and "ASC or AGC" cytology

\begin{tabular}{|c|c|c|c|c|c|c|}
\hline Cytology & HPV test & Total & zHSIL & Odds ratio & $95 \% \mathrm{Cl}$ & $p$-value \\
\hline \multirow[t]{7}{*}{$\overline{N I L M}$} & HPV DNA chip & & & & & \\
\hline & HR-HPV (-) & 186 & $15(8.1)$ & & & \\
\hline & Non-16/18 HR-HPV (+) & 69 & $7(10.1)$ & 2.119 & $0.642-6.995$ & .217 \\
\hline & HPV-16/18 (+) & 67 & 18 (26.9) & 6.756 & $2.114-21.588$ & .000 \\
\hline & HPV HC2 & & & & & \\
\hline & HR-HPV (-) & 227 & $14(6.2)$ & & & \\
\hline & HR-HPV (+) & 95 & $26(27.4)$ & 5.894 & $2.852-12.180$ & .000 \\
\hline \multirow[t]{7}{*}{ ASC or AGC } & HPV DNA chip ${ }^{a}$ & & & & & \\
\hline & HR-HPV (-) & 52 & $6(11.5)$ & & & \\
\hline & Non-16/18 HR-HPV (+) & 59 & $13(22.0)$ & 2.454 & $0.819-7.353$ & .190 \\
\hline & HPV-16/18 (+) & 63 & 35 (55.6) & 11.715 & $4.101-33.463$ & .000 \\
\hline & HPV HC2 & & & & & \\
\hline & HR-HPV (-) & 50 & $8(16.0)$ & & & \\
\hline & HR-HPV (+) & 124 & $46(37.1)$ & 2.988 & $1.269-7.039$ & .012 \\
\hline
\end{tabular}

$\geq \mathrm{HSIL}$, high-grade squamous intraepithelial lesion or worse; HPV, human papillomavirus; NILM, negative for intraepithelial lesion or malignancy; ASC, atypical squamous cells; AGC, atypical glandular cells; Cl, confidence interval; HR, high-risk; HC2, Hybrid Capture 2.

aHR-HPV (-) includes HPV (-) or low-risk HPV (+) or "HPV-other types" (+); non-16/18 HR-HPV (+) includes HPV-16 (-) and HPV-18 (-), 13 other HR-HPV (+) with or without low-risk HPV (+) or "HPV-other types" (+); HPV-16/18 (+) includes HPV-16 (+) and/or HPV-18 (+), with or without any other HPV genotypes present. 
non-16/18 HR-HPV (+) group did not significantly differ from the OR in the HR-HPV (-) group. Based on the HC2 results, the age-adjusted OR for $\geq \mathrm{HSIL}$ was 3.0 (95\% CI, 1.3 to 7.0 ) in the HR-HPV (+) group (Table 5).

\section{DISCUSSION}

HPV-16 and HPV-18 are the main carcinogenic HPV genotypes associated with cervical cancer and are responsible for $55 \%-60 \%$ and $10 \%-15 \%$ of invasive cancers, respectively. Non-16/18 HR-HPV genotypes are associated with 25\%-35\% of invasive cancers. ${ }^{1,711}$ Because the importance of HPV-16 and HPV-18 in cervical pathology has been sufficiently demonstrated, the updated U.S screening guidelines for the early detection of cervical cancer and its precursors recommend co-testing (cytology in combination with HR-HPV testing) over cytology alone and integrated HPV-16/18 genotyping for the management of patients whose HPV test was positive and cytology negative. ${ }^{8}$ Recently, interim guidelines stating that primary HRHPV testing for cervical cancer screening can be considered an alternative to current U.S cervical cancer screening methods have been published. ${ }^{12}$

In many Asian countries, including Korea, the HPV DNA chip test and PCR-based HPV detection kits as well as the HC2 test are commonly used in clinical settings because the sensitivity and specificity of the HPV DNA chip test and PCR-based HPV detection kits are comparable to the HC2 test for the detection of HSIL or worse disease. ${ }^{13}$ The concordance rate between the HPV DNA chip and the HC2 test was previously reported to be $88 \%$ ( $\kappa=0.61) .{ }^{14}$ In our study, the concordance rate was $79.4 \%$ (688/867), with a $\kappa$-value of 0.55 . The HPV DNA chip exhibits concordance rates ranging from $61.5 \%$ and $91.1 \%$ compared with DNA sequencing. ${ }^{15,16}$ The wide range of concordance rates for the HPV DNA chip compared with other assays may result from different viral detection thresholds and crossreactivity. The sensitivity of the HC2 method is approximately 5,000 copies of the HPV genome. ${ }^{17}$ The PCR-amplification HPV DNA chip method is subject to a minimum signal detection level, which is the scanner signal:background noise ratio limit at low viral loads, approximately 100 to 1,000 copies. ${ }^{18}$ Additionally, a simple primer set targeting the HPV MY09 region and sequence similarities between the 24 probes of the HPV DNA chip may lead to cross-reactivity that contributes to discordance. ${ }^{16,19}$ In this study, the clinical performance of the MyHPV chip was comparable to HC2 for detecting HSILs or worse. The sensitivity and specificity of both HPV tests and cy- tology were very similar (approximately $85 \%$ and $50 \%$, respectively). Adding the HPV test (MyHPV chip or HC2) to cytology exhibited excellent sensitivity (95\%, each) but low specificity (approximately 30\%, each). Furthermore, we demonstrated that patients who were HPV-16/18 (+) according to the MyHPV chip were at a higher risk of lesions graded HSIL or worse than patients who were non-16/18 HR-HPV (+) (OR 9.9 vs. 3.7). Among cytology "NILM" and "ASC or AGC" subgroups, the ORs were 5.9 and 3.0, respectively, for patients HR-HPV (+) based on HC2 test, compared with HR-HPV (-) patients. Conversely, patients who tested HR-16/18 positive using the MyHPV chip showed much higher ORs (6.8 and 11.7, respectively). According to these results, clinicians need to refer patients who are HPV-16/18 (+) according to HPV genotyping for immediate colposcopy instead of follow up, even when the cytology test is negative or ambiguous.

We found that patients who were non-16/18 HR-HPV (+) according to the MyHPV chip showed higher risk for $\geq$ HSILs than patients with HR-HPV (-) (OR, 3.7). However, in the subgroup study of cytology "NILM" and "ASC or AGC," the OR for $\geq$ HSILs in the non-16/18 HR-HPV (+) group did not significantly differ from the HR-HPV (-) group, probably due to the small subgroup population.

The limitations of our study include its retrospective design and that it was conducted at a single institution. Additionally, our patients were primarily referred from local clinics and previously diagnosed as "abnormal" based on local cervical cytology. The overall prevalence rates of $\geq$ HSIL in NILM (12.4\%) and ASCUS (25.4\%) patients were higher than those reported in a previous study. ${ }^{4}$ We hypothesize the cytology may have been under sampled because clinicians tend to forego subsequent extensive sampling if previously diagnosed.

In conclusion, the sensitivity and specificity of the HPV DNA chip test are similar to the HC2 test, and detecting HPV 16/18 with a HPV DNA chip is useful for predicting high-grade cervical lesions. Therefore, the HPV DNA chip genotyping method, which is commonly used in many Asian countries as an HPV DNA test, may be useful in assessing the risk of high-grade cervical lesions.

\section{Conflicts of Interest}

No potential conflicts of interest relevant to this article are reported. 


\section{REFERENCES}

1. Walboomers JM, Jacobs MV, Manos MM, et al. Human papillomavirus is a necessary cause of invasive cervical cancer worldwide. J Pathol 1999; 189: 12-9.

2. Castle PE, Solomon D, Schiffman M, Wheeler CM. Human papillomavirus type 16 infections and 2-year absolute risk of cervical precancer in women with equivocal or mild cytologic abnormalities. J Natl Cancer Inst 2005; 97: 1066-71.

3. Khan MJ, Castle PE, Lorincz AT, et al. The elevated 10-year risk of cervical precancer and cancer in women with human papillomavirus (HPV) type 16 or 18 and the possible utility of type-specific HPV testing in clinical practice. J Natl Cancer Inst 2005; 97: 1072-9.

4. Stoler MH, Wright TC Jr, Sharma A, et al. High-risk human papillomavirus testing in women with ASC-US cytology: results from the ATHENA HPV study. Am J Clin Pathol 2011; 135: 468-75.

5. Wright TC Jr, Stoler MH, Sharma A, et al. Evaluation of HPV-16 and HPV-18 genotyping for the triage of women with high-risk HPV+ cytology-negative results. Am J Clin Pathol 2011; 136: 578-86.

6. Bosch FX, Burchell AN, Schiffman M, et al. Epidemiology and natural history of human papillomavirus infections and type-specific implications in cervical neoplasia. Vaccine 2008; 26 Suppl 10: K1-16.

7. de Sanjose S, Quint WG, Alemany L, et al. Human papillomavirus genotype attribution in invasive cervical cancer: a retrospective cross-sectional worldwide study. Lancet Oncol 2010; 11: 1048-56.

8. Saslow D, Solomon D, Lawson HW, et al. American Cancer Society, American Society for Colposcopy and Cervical Pathology, and American Society for Clinical Pathology screening guidelines for the prevention and early detection of cervical cancer. Am J Clin Pathol 2012; 137: 516-42.

9. Solomon D, Davey D, Kurman R, et al. The 2001 Bethesda System: terminology for reporting results of cervical cytology. JAMA 2002; 287: 2114-9.
10. Terry G, Ho L, Londesborough P, Cuzick J, Mielzynska-Lohnas I, Lorincz A. Detection of high-risk HPV types by the Hybrid Capture 2 test. J Med Virol 2001; 65: 155-62.

11. Muñoz N, Bosch FX, de Sanjosé S, et al. Epidemiologic classification of human papillomavirus types associated with cervical cancer. $\mathrm{N}$ Engl J Med 2003; 348: 518-27.

12. Huh WK, Ault KA, Chelmow D, et al. Use of primary high-risk human papillomavirus testing for cervical cancer screening: interim clinical guidance. Obstet Gynecol 2015; 125: 330-7.

13. Lee JK, Hong JH, Kang S, et al. Practice guidelines for the early detection of cervical cancer in Korea: Korean Society of Gynecologic Oncology and the Korean Society for Cytopathology 2012 edition. J Gynecol Oncol 2013; 24: 186-203.

14. Kang WD, Kim CH, Cho MK, et al. Comparison of the hybrid capture II assay with the human papillomavirus DNA chip test for the detection of high-grade cervical lesions. Int J Gynecol Cancer 2009; 19: 924-8.

15. Choi YD, Jung WW, Nam JH, Choi HS, Park CS. Detection of HPV genotypes in cervical lesions by the HPV DNA Chip and sequencing. Gynecol Oncol 2005; 98: 369-75.

16. Park S, Kang Y, Kim DG, Kim EC, Park SS, Seong MW. Comparison of the analytical and clinical performances of Abbott RealTime High Risk HPV, Hybrid Capture 2, and DNA Chip assays in gynecology patients. Diagn Microbiol Infect Dis 2013; 76: 432-6.

17. Brink AA, Snijders PJ, Meijer CJ. HPV detection methods. Dis Markers 2007; 23: 273-81.

18. Um TH, Lee EH, Chi HS, Kim JW, Hong YJ, Cha YJ. Comparison of HPV genotyping assays and Hybrid Capture 2 for detection of highrisk HPV in cervical specimens. Ann Clin Lab Sci 2011; 41: 48-55.

19. Cho EJ, Do JH, Kim YS, Bae S, Ahn WS. Evaluation of a liquid bead array system for high-risk human papillomavirus detection and genotyping in comparison with Hybrid Capture II, DNA chip and sequencing methods. J Med Microbiol 2011; 60 (Pt 2): 162-71. 